\title{
Finite Element Modeling of Mulliscale Transport Phenomena
}


This page intentionally left blank 


\title{
Finite Element Modeling of Multiscale Transport Phenomena
}

\author{
Vahid Nassehi \\ Loughborough University, UK \\ Mahmoud Parvazinia \\ Iran Polymer and Petrochemical Institute, Iran
}

Imperial College Press 
Published by

Imperial College Press

57 Shelton Street

Covent Garden

London WC2H 9HE

\section{Distributed by}

World Scientific Publishing Co. Pte. Ltd.

5 Toh Tuck Link, Singapore 596224

USA office: 27 Warren Street, Suite 401-402, Hackensack, NJ 07601

UK office: 57 Shelton Street, Covent Garden, London WC2H 9HE

\section{British Library Cataloguing-in-Publication Data}

A catalogue record for this book is available from the British Library.

\section{FINITE ELEMENT MODELING OF MULTISCALE PHENOMENA}

Copyright $\left({ }^{\circ} 2011\right.$ by Imperial College Press

All rights reserved. This book, or parts thereof, may not be reproduced in any form or by any means, electronic or mechanical, including photocopying, recording or any information storage and retrieval system now known or to be invented, without written permission from the Publisher.

For photocopying of material in this volume, please pay a copying fee through the Copyright Clearance Center, Inc., 222 Rosewood Drive, Danvers, MA 01923, USA. In this case permission to photocopy is not required from the publisher.

Desk Editor: Tjan Kwang Wei

ISBN-13 978-1-84816-429-1

ISBN-10 1-84816-429-7

Typeset by Stallion Press

Email: enquiries@ stallionpress.com

Printed in Singapore. 
For our families 
This page intentionally left blank 


\section{Preface}

For more than five decades finite element method has been regarded as the foremost numerical technique for the solution of governing equations of engineering problems. Using this method powerful computer schemes, which combine mathematical rigor with geometrical flexibility in dealing with complex problems, have been developed. These schemes are, in general, the most reliable predictive simulation tools available to researchers and design engineers. Inherent flexibility and sound mathematical basis of the technique has been the main reason for its progress and evolution over the years, enabling numerical analysts to use the technique to solve a wide variety of problems. Recent advances in the power and capability of computer systems has made simulation of some of the most complex physical situations a realistic possibility. However, novel concepts need to be incorporated into existing numerical schemes in order to take advantage of recent advances in low cost computing. In this respect, the solution of multiscale phenomena, in which significant and rapid variations in the behavior of field unknowns either precludes the application of traditional techniques or renders their use complicated and unyielding, has been the subject of intense research during the past decade. During this period many important research papers have appeared which provide robust mathematical foundations for the construction of practical finite element schemes for multiscale problems.

The main focus of this book is to provide a simple to follow account of the development of a class of practical multiscale weighted residual finite element schemes for field problems encountered in fluid flow and transport processes. In particular, dealing with the generic multiscale phenomena which affect the design and analysis of chemical engineering and polymer processing operations has been our objective.

The book starts with an explanation of the weighted residuals finite element technique to provide the necessary background for the discussions presented later on in the book. Readers who have not previously used weighted 
residuals finite element schemes should, nevertheless, be able to follow the discussions presented in chapters dealing with the extension of this technique to multiscale problems. Almost all of the topics introduced in the text have been supplemented with solved examples. These examples can be used as a guide by readers to apply the constructed schemes to their own problems or they may use the described methodology for the development of multiscale schemes applicable to other problems.

Finally, we have included a detailed listing of the computer code used to solve many of the examples given in this book and provided sample input and output files. Readers can repeat the illustrated examples and gain experience for extending the program to perform their own multiscale finite element simulations.

\author{
Vahid Nassehi \\ Mahmoud Parvazinia
}




\section{Contents}

1. Weighted Residual Finite Element Method 1

1.1 Basic Concept . . . . . . . . . . . . . . . . . . . . 1

1.1.1 Practical procedure . . . . . . . . . . . 2

1.1.2 Finite element approximations . . . . . . . . . 4

1.2 Numerical Integration $\ldots \ldots \ldots$

1.2.1 Mapping of irregular and curved elements onto master elements . . . . . . . . . . . 7

1.3 Steps Used to Obtain a Finite Element Solution for a Field Problem . . . . . . . . . . . . . . . . . . 13

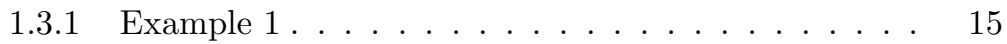

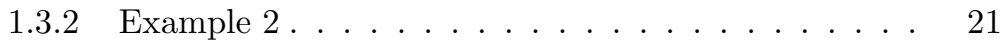

References........................ 27

2. Shape Functions and Fundamental Properties of Finite Elements $\quad 29$

2.1 Interpolation Polynomials . . . . . . . . . . . . . . 30

2.2 Shape Functions of Simplex Elements . . . . . . . . . . . 31

2.2.1 One-dimensional linear element . . . . . . . . . . 31

2.2.2 Two-dimensional simplex element . . . . . . . . . 32

2.2.3 Three-dimensional simplex element . . . . . . . . 35

2.3 Some Examples of Complex and Multiplex Elements . . . . 37

2.3.1 Quadratic and cubic one-dimensional elements ... 37

2.3.2 Four-node two-dimensional element . . . . . . . . . 40

2.4 Convergence of Finite Element Approximations . . . . . . . 42

2.5 Continuity Conditions . . . . . . . . . . . . . . . 44

2.6 Solved Examples . . . . . . . . . . . . . . . . . . 44

2.6.1 Example 1: Limitation of the standard Galerkin procedure . . . . . . . . . . . . 4 44 
2.6.2 Example 2: A further problem associated with the use of the standard Galerkin finite element method .

2.6.3 Bubble function enriched elements . . . . . . . . 51

References ...................... 53

\section{Basic Concepts of Multiscale Finite Element Modeling}

3.1 Stabilization of Finite Element Schemes . . . . . . . . . 55

3.1.1 Upwinded finite element schemes . . . . . . . . . . . . 56

3.1 .2 Upwinding techniques . . . . . . . . . . . 57

3.2 Multiscale Approach . . . . . . . . . . . . . . . . 59

3.2.1 Variational multiscale method . . . . . . . . . 60

3.2 .2 (RFB) function method . . . . . . . . . . 62

3.3 Practical Implementation of the RFB Function Method . . 64

3.3.1 Multiscale finite element solution of the DR equation using RFB function method . . . . . . . . . . 65

3.3.2 Multiscale finite element solution of the CD equation using RFB function method . . . . . . . 87

3.3.3 Multiscale finite element solution of the CDR equation using RFB function method . . . . . . . . 94

3.4 Practical Implementation of the STC Method . . . . . . . . 106

3.4.1 Multiscale finite element solution of the DR equation using STC method . . . . . . . . . . . . 108

3.4.2 Multiscale finite element solution of the CD equation using STC method . . . . . . . . . . . . . . . 113

3.4.3 Multiscale finite element solution of the CDR equation using STC method . . . . . . . . . . . 118

References . . . . . . . . . . . . . . . . . . 122

\section{Simulation of Multiscale Transport Phenomena} in Multidimensional Domains

4.1 Two-dimensional Multiscale Finite

Element Technique . . . . . . . . . . . . . . 125

4.2 Selection of Bubble Functions and Calculation of Bubble Coefficients . . . . . . . . . . . . . . . 129

4.3 RFB Functions Corresponding to the Two-Dimensional DR Equation . . . . . . . . . 131

4.3.1 Derivation of polynomial bubble function coefficients for the DR equation using the STC method . . . . . 133

4.3.2 Elimination of the boundary integrals . . . . . . . 133 
4.3.3 Solution of a benchmark two-dimensional

DR problem . . . . . . . . . . . . . . . 134

4.4 Solution of Two-Dimensional Convection-Diffusion (CD) Equation . . . . . . . . . . . . . . . . 140

4.4.1 Governing CD equation and boundary conditions . . 140

4.4.2 RFB functions corresponding to the two-dimensional CD equation . . . . . . . . 141

4.4.3 Two-dimensional bubble functions for bilinear elements . . . . . . . . . . . . . . . . . 141

4.4.4 Elimination of the boundary integrals . . . . . . . . 142

4.4.5 Solution of a benchmark two-dimensional CD problem . . . . . . . . . . . . . . . . 143

4.5 Solution of Convection-Diffusion-Reaction

(CDR) Problems . . . . . . . . . . . . . . . . . . 143

4.5.1 Governing CDR equation and boundary conditions . . . . . . . . . . . . . . . . . 144

4.5.2 Derivation of RFB functions for the CDR equation . . . . . . . . . . . . 146

4.5.3 Solution of a benchmark two-dimensional CDR equation . . . . . . . . . . . 146

4.6 Solution of Transport Equations Using Bubble Function-Enriched Triangular Elements . . . . . . . . . . 149

4.7 Multiscale Finite Element Methods for Time-Dependent Problems . . . . . . . . . . . . . . . . . 152

4.7.1 Multiscale space-time finite element discretization . . . . . . . . . . . . 153

4.7.2 Multiscale space-time finite element modeling . . . . 154

4.7.3 Two-dimensional space-time bubble functions for enrichment of bilinear elements . . . . . . . 155

4.7.4 Elimination of the boundary integrals . . . . . . 156

4.7.5 Transient CD problem . . . . . . . . . . . 156 References . . . . . . . . . . . . . . . . 158

\section{Application of Multiscale Finite Element} Schemes to Fluid Flow Problems

5.1 Two-Dimensional and Axisymmetric Flow Regimes Governing Equations . . . . . . . . . . . . . . . . 161 
5.2 Modeling of Isothermal Brinkman Flow of a Newtonian Fluid in a Two-Dimensional Domain Using Multiscale Finite Element Schemes . . . . . . . . . . . . . . . . . . . 164

5.2 .1 Continuous penalty scheme . . . . . . . . 167

5.2.2 Bubble-enriched shape functions used in conjunction with the continuous penalty method . . . . . . . . 172

5.2.3 Solved example — unidirectional flow . . . . . . . 172

5.3 Solution of the Energy Equation . . . . . . . . . . . . 174

5.3.1 Finite element scheme used to solve the energy equation . . . . . . . . . . . 175

5.3.2 Bubble-enriched shape functions used in the solution of the energy equation . . . . . . . . . . . 176

5.3.3 Solved example - Non-isothermal unidirectional flow . . . . . . . . . . . 176

5.3.4 Higher-order elements and mesh refinement . . . . . 179

5.4 Multidirectional, Nonisothermal Porous Flow . . . . . . . . 180

5.5 Inclusion of Inertia Effects in Porous Flow Models . . . . . 181

5.5.1 Derivation of bubble functions for Brinkman-HazenDupuit-Darcy equation . . . . . . . . . . . . 184

5.5.2 Solved example - High velocity unidirectional porous flow . . . . . . . . . . . . . . . 184

5.5.3 Solved example - Multidirectional, high-velocity porous flow . . . . . . . . . . . . . 185

5.6 Solution of Axisymmetric Brinkman Equation . . . . . . . . 185

5.6.1 Derivation of the continuous penalty finite element scheme for axisymmetric Brinkman equation . . . . 188

5.6.2 Derivation of bubble functions for axisymmetric Brinkman equation . . . . . . . . . . . . . 189

References . . . . . . . . . . . . . . . . . . . 191

6. Computer Program 193

6.1 Program Structure . . . . . . . . . . . . . . . . . . . . 193

6.2 Source Code . . . . . . . . . . . . . . . . . . . . . 194

6.3 Input File Structure . . . . . . . . . . . . . . . . . . 220

6.4 Output Files . . . . . . . . . . . . . . . . . 225

6.4.1 DR equation — dissipation case . . . . . . . 225

6.4.2 DR equation — production case . . . . . . . . 228 
6.4 .3 CD equation . . . . . . . . . . . . . 230

6.4.4 CDR equation ................ 233

$\begin{array}{ll}\text { Appendices } & 237\end{array}$

$\begin{array}{ll}\text { Author Index } & 245\end{array}$

$\begin{array}{ll}\text { Subject Index } & 247\end{array}$ 